\title{
Surface texture evaluation of additively manufactured metallic cellular scaffolds for acetabular implants using X-ray computed tomography
}

\author{
Shan Lou ${ }^{1} \cdot$ Luca Pagani $^{1} \cdot$ Wenhan Zeng ${ }^{1} \cdot$ Muhammad U. Ghori ${ }^{2} \cdot$ Xiangqian Jiang $^{1} \cdot$ Paul J. Scott $^{1}$
}

Received: 17 April 2019 / Accepted: 4 May 2019 / Published online: 22 May 2019

(c) The Author(s) 2019

\begin{abstract}
The surface topography of acetabular implants plays a key role in providing cell attachment and proliferation. The measurement and characterisation of the surface texture of the cellular scaffold layer on the acetabular cup are very difficult due to the 3D nature of scaffold geometry. It is proposed to use X-ray computed tomography (XCT) to measure the surface texture of an electron beam melting-produced titanium acetabular cup. The surface texture of its cellular scaffold is evaluated using the newly developed 3D surface texture parameters, which allows surface characterisation on 3D triangular mesh surfaces. Four commonly used height parameters, i.e. the arithmetical mean height $\mathrm{Sa}$, the root mean square height $\mathrm{Sq}$, the skewness Ssk and the kurtosis Sku, are calculated from surface patches extracted from the XCT scanned triangular mesh surface. In addition, the surface peak density and pit density, which are more related to cell communication and proliferation, are estimated based on the 3D watershed segmentation. The Wolf pruning with an empirical threshold $12 \mu \mathrm{m}$ is used to control the over-segmentation.
\end{abstract}

Keywords Additive manufacturing $\cdot$ Cellular scaffold $\cdot$ Surface texture $\cdot$ X-ray computed tomography

\section{Introduction}

By adding materials in layers directly from digital models, additive manufacturing (AM) technology offers a number of advantages over conventional (subtractive) manufacturing techniques. One of the major advantages over traditional manufacturing processes is the AM's capability of constructing freeform geometries. This led to the wide employment of AM technology in healthcare applications, particularly in creating scaffolds for medical implants [1]. AM enables accurate patient-specific implants to be created, using the patient's X-ray computed tomography (XCT) or magnetic resonance image (MRI) scan data, allowing implants to be personalised to match each patient's individual needs. In addition, cellular scaffold structures for orthopaedic

Shan Lou

s.lou@hud.ac.uk

1 EPSRC Future Metrology Hub, University of Huddersfield, Queensgate, Huddersfield HD1 3DH, UK

2 Department of Pharmacy, University of Huddersfield, Queensgate, Huddersfield HD1 3DH, UK implants can be easily made via AM, which on the contrary are almost impossible to be crafted using traditional manufacturing methods.

In comparison with solid metal materials, open-cellular scaffolds of the in-growth layers of joint implants, as nowadays adopted by many healthcare companies, are proved to be more effective structures to support cell attachment and proliferation, cell morphology and spreading as well as better bone-implant fixation [2]. See Fig. 1 for an acetabular implant sample produced by Arcam's electron beam melting (EBM) technology. These metallic interconnected cellular structures have an optimal combination of surface roughness, pore size and porosity [3, 4]. Rough surface texture can promote cell attachment and proliferation [5]; good biocompatibility can be achieved by using bio-friendly metal materials, e.g. titanium and its alloys; suitable pore morphology and porosity can mimic the structure of native bone to promote cell in-growth and provide channels for nutrient and waste transportation [6]; cellular structure can reduce the weight of implants while keeping necessary mechanical properties and, in addition, alleviate the mechanical mismatch between metallic implant and natural bone, which 


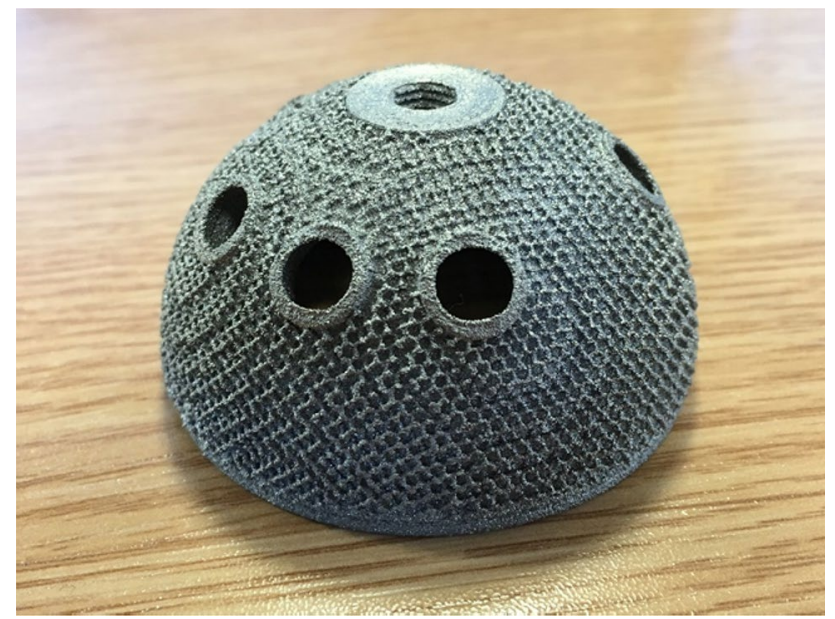

Fig. 1 An acetabular cup with cellular scaffold for improved osseointegration

might cause bone resorption and eventually the failure of metallic implant fixation [7].

\section{Impacts of surface quality of metallic AM implants}

Surface topography of implant not only affects the boneimplant contact, but also the biomechanical interaction of that interface at early implantation periods [8]. Rough surfaces have found widespread application in implantology. The as-built SLM and EBM surfaces have rough surface textures with Ra values ranging from several to tens of microns. The raw surface topography produced by AM tends to favour cell proliferation, because these surfaces often present small bumps, surface pores and 3D re-entrant features which can enhance bone-to-implant contact and also provide strong bonding capability similar to the mechanical interlocking mechanism [9]. The frictional and bone in-growth properties of EBM-produced surface structure were found to be comparable, and after 15 weeks better than that of the plasmasprayed surface [3]. No large discrepancy was observed between the trabecular bone implants made by EBM and SLM processes, even though their gross morphology and surface texture are different due to different processing conditions [10].

There is a controversy on whether post-processing is required for surface treatment of AM implants, however. The advocators of surface treatment found that with surface modification, e.g. sand blasting and acid etching, implants behave much better in terms of bone formation and bridging [11]. Jolota et al. [12] observed that the titanium samples treated by supersaturated calcium phosphate $(\mathrm{CaP})$ solutions have a double rate of cell proliferation in comparison with neat surfaces and even actin cytoskeleton and stress fibres became visible. However, on the opposite side, other researchers reported that not much improvements were achieved through surface post-processing. Biemond et al. found the presence of calcium phosphate coating to SLM and EBM specimens did not improve the bone-implant contact and did not produce better bone in-growth or mechanical fixation [10]. No significant difference in the bone in-growth with and without apatite coating was noticed by Li et al. [4], which suggested AM-produced implants can be directly used without modification. A possible explanation to this controversy was suggested by Sing et al. [13] that surface roughness in the specific range is favourable for osseointegration. It was found that the surface roughness of EBM implants with Ra below $24.9 \mu \mathrm{m}$ has a positive effect on proliferation and differentiation of human osteoblasts, while roughness above $56.9 \mu \mathrm{m}$ has a negative impact on the proliferation.

The surface quality of scaffold structure must be taken extra care to avoid too much high surface peaks and scarped flanks, since these steep geometries can inhibit cell-cell interactions and thus hinder cell proliferation; in comparison, surface topography with dense pits can favour cell proliferation in that cells prefer lying the concave hollows of the matrix as this behaviour is associated with a low-energy expenditure [14]. Thus, the distance between peaks and the quality of the peaks (sharp or round) are of concerns for the success of a biomaterials [15].

\section{Surface measurement of metallic AM implants}

As aforementioned, surface quality is of critical importance to osseointegration of implants. Only surfaces with preferred surface roughness combined with optimised micro-geometry (e.g. pits) can bring to better bone tissue in-growth. Surface roughness measurement has been highlighted in the literature, which is often performed using tactile and optical measurement sensors. For instance, Biemond et al. [10] employed a Universal Surface Tester with a tactile stylus to examine surface roughness of porous implant specimens produced by EBM; Ponader et al. [14] used a KORAD white-light interferometry to measure their titanium cellular implant samples; Li et al. [4] used the optical profiler (Wyko NT9300) to inspect the top and lateral surface of porous Ti6Al4V samples. All these works presented the quantitative results of surface quality using surface texture parameters, more precisely, the height parameters, either profile parameters, e.g. $\mathrm{Ra} / \mathrm{Rq}$ or areal parameters, e.g. $\mathrm{Sa} / \mathrm{Sq}$.

A fatal challenge of surface measurement of scaffold implant, however, was identified by Biemond et al. [3], who claimed that it appeared to be impossible to define the surface roughness of the porous structures accurately because of their three-dimensional character. Due to the layer-bylayer nature of AM processes, the produced surface texture varies depending on the part orientation as well as other relevant process variables, e.g. layer thickness. The top, 
bottom and lateral parts of the strut surface can present different surface topography. This was evidenced by $\mathrm{Li}$ et al. [4]. The top surfaces of their porous titanium samples have a relatively smooth surface with Ra 5-10 $\mu$ m, while the lateral surfaces appear much rougher with plenty of surface bumps and cavities (Ra 15-21 $\mu \mathrm{m})$. The measurements were done on each separate portion, which is planar or nearly planar. A follow-on challenge is the selection of appropriate surface parameters for the characterisation of porous surface in terms of osseointegration. Currently, only the height parameters, e.g. Ra, Rq, Rp, Rk, or their areal counterparts, were adopted to quantify the surface quality. However, it was suggested that the micro-geometry on the surface, e.g. surface peaks and pits, is playing a major role for cell attachment and tissue growth [14]. Apart from the characterisation of surface texture, a fundamental obstacle is surface measurement technique itself. Current optical and tactile techniques are not able to measure internal surfaces of cellular structures, whose intricate forms do not permit line-of-sight [16]. It is noticed that in current literature only external surfaces of acetabular implants were measured. The examination of internal surface quality without physically breaking the part has not been found.

In this paper, a novel methodology to evaluate the surface quality of additively manufactured metallic cellular scaffold for acetabular implant using XCT is presented. Particularly, three challenges addressed above will be dealt by using 3D feature-based surface texture parameters coupled with the employment of X-ray computed tomography (XCT) for surface measurement. The paper is organised as follows: Sect. 2 describes the basic principle of XCT, the feasibility of using $\mathrm{XCT}$ to measure AM surface texture and the set-up of XCT scanning to measure an acetabular cup; Sect. 3 addresses the evaluation of 3D surface texture of cellular scaffold; a discussion of the physical XCT scanning as well as the parameterisation of cellular scaffold surface is followed in Sect. 4; the paper is concluded in Sect. 5.

\section{XCT measurement of surface texture}

\section{Basic principle of XCT measurement}

The XCT measurement relies on the object's material absorption of high-energy radiation. An X-ray source emits radiation, passing through the object under measurement and captured by an X-ray detector [17]. As shown in Fig. 2, a sequence of projection is generated from rotating either the X-ray source or the measurement object, resulting in a sequence of 2D greyscale image slices [18]. Thereafter, the $3 \mathrm{D}$ volumetric data are reconstructed as a cube of 3D voxels, each voxel is associated with a grayscale intensity. The

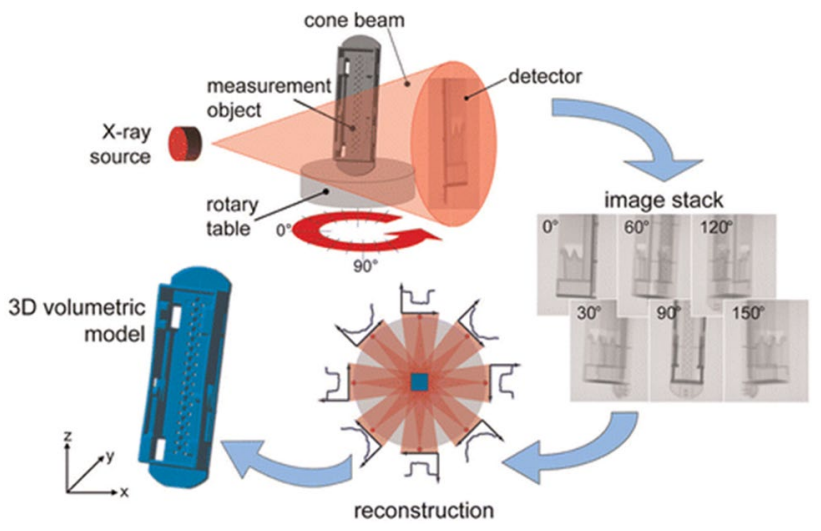

Fig. 2 Basic principle of XCT in dimensional metrology [18]

intensity $I$ indicates the remaining radiation after material absorption and is obtained by Lambert-Beer's law (Eq. 1),

$I=I_{0} \cdot e^{-\mu d}$

where $I_{0}$ is the original X-ray intensity, $d$ is the material thickness and $\mu$ is the absorption coefficient which is associated with the atomic number and density of materials.

To perform the dimensional measurements of geometrical quantities, those voxels representing the boundary of materials or the surface of the measured object are extracted out from the volumetric data. It is normally done by setting a global thresholding based on the information of the greyvalue histogram. For example, the ISO 50 method takes the threshold as the average of the material peak grey value and the background peak grey value. This threshold value is then used to differentiate the material voxels from the background voxels. More advanced local adaptive methods are nowadays available to distinguish between the object materials and the air [16, 19-21]. The boundaries of the volume representing the surfaces are converted into a polygonal mesh using the marching cubes algorithm [22] or its variants.

\section{Surface texture measurement by XCT}

Originated as an inspection tool for medical applications, XCT has been increasingly used for industrial purposes, particularly as a non-destructive method for measuring porosity and metrology tool for examining the dimensions of products with complex geometries. In comparison with the widely used tactile and optical measurement techniques, XCT does not have the limitation on accessing complex external and internal geometries, thus it is quite suitable for the measurement of AM components, e.g. cellular scaffold structures.

In recent years, using XCT for surface texture measurement began to attract researchers' attention, even though the use of XCT for this purpose is still restricted due to the 
limited resolution of XCT physical system as well as the data analysis capability of its software. Researchers from the KU Leuven University illustrated a comparison study on using tactile, optical and micro-focus XCT methods to measure AM components with different surface roughness [23]. In the case of higher roughness (micro-scale) surfaces, the roughness result $(\mathrm{Ra})$ of micro-focus XCT was comparable to the tactile profilometer. Townsend et al. [24] compared focus variation technique with XCT and a good agreement was found. They also took surface determination and filament into consideration and found that Sa resulted from XCT before and after the filament change was $1.8 \%$ and $2.7 \%$ higher than that of focus variation. Thompson et al. [25] illustrated a comparison study of using three optical systems and one XCT system to measure the internal surface texture of a direct metal laser sintering (DMLS) artefact and found that $\mathrm{Sa}$ and $\mathrm{Sq}$ generated by XCT measurement are similar to those of confocal microscopy and coherence scanning interferometry.

\section{XCT scanning set-up}

A Nikon MCT225 device was used to scan a titanium acetabular cup testing sample (diameter: $\sim 60 \mathrm{~mm}$ ) made by EBM. The in-chamber set-up of XCT scanning is illustrated in Fig. 3. The acetabular sample was placed on a polymeric foam, sitting on a rotary stage. The stage was moved close to the X-ray gun in order to get the magnification as large as possible. X-ray radiation was fired by the gun and penetrated through the acetabular sample. The residual radiation was received by the detector to generate the projection images. The key parameters for XCT scanning are listed in Table 1.

The whole scanning took $104 \mathrm{~min}$. A total of 3141 projection images were then reconstructed into a $3 \mathrm{D}$ volume using Nikon's CTPro 3D software. The follow-on data analysis of the 3D volumetric data was dealt via the VGStudio Max 2.2 [26]. The advanced local iterative algorithm was used to find the material boundary based on the local surrounding voxel. A local threshold method can significantly compensate the deviation caused by the acquisition issues, such as

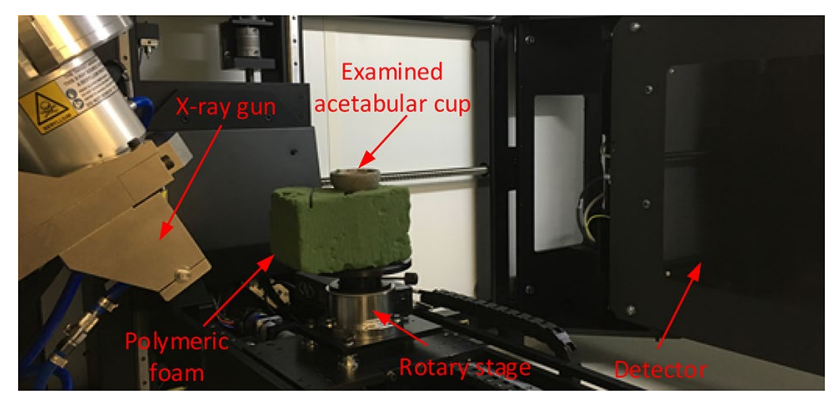

Fig. 3 In-chamber set-up of XCT scanning for the titanium acetabular cap
Table 1 Scanning parameters of Nikon XCT $225 \mathrm{M}$

\begin{tabular}{ll}
\hline XCT scanning parameters & Values \\
\hline Beam energy & $170 \mathrm{kV}$ \\
Beam current & $118 \mu \mathrm{A}$ \\
Power & $20.4 \mathrm{~W}$ \\
Filter & Copper, $1 \mathrm{~mm}$ \\
Detector size & $2000 \times 2000$ pixels \\
Voxel size & $31.7 \mu \mathrm{m}$ \\
Number of projections & 3141 \\
\hline
\end{tabular}

beam hardening. Figure 4 illustrates the reconstructed surface model of the acetabular cup. The cup surface data were then exported as stereo-lithography (STL) mesh format, using the VGStudio Max 2.2 "Super Precise" setting, which provides the highest available resolution, i.e. the mesh simplification was not performed. The mesh was then processed using RameshCleaner [27] to remove non-manifold edges, and it was finally remeshed using the anisotropic algorithm proposed by Botsch et al. [28]. A magnification showing the differences before and after the remeshing is illustrated in Fig. 5. The mesh extracted from the volume presents elongated triangles, while the edges' lengths of the triangles of the processed mesh are similar.

\section{Surface texture evaluation}

Surface texture is usually evaluated by computing a set of indexes giving information about certain features of the surface or its variability in comparison with a reference. Due to the complexity of the 3D cellular scaffold structure on the

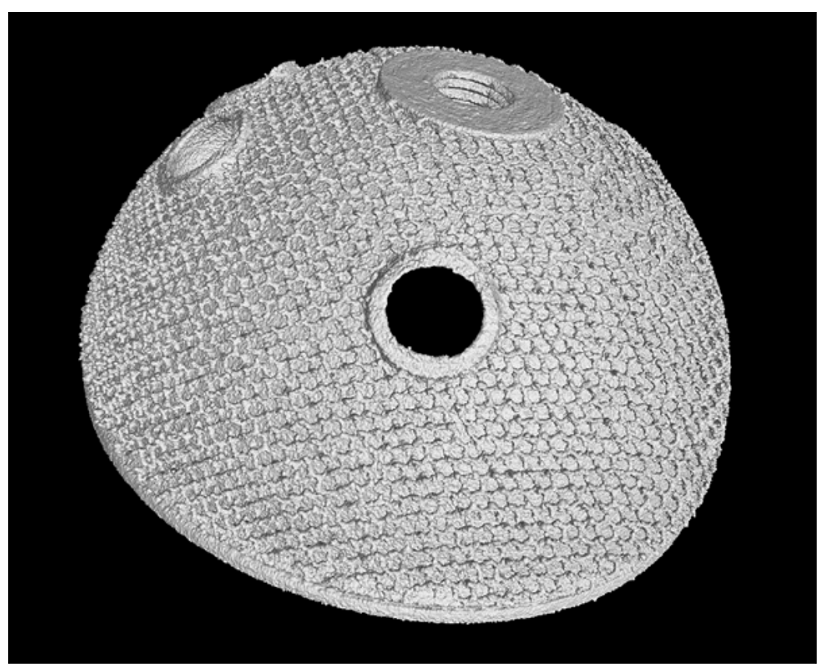

Fig. 4 Reconstructed 3D surface of the titanium acetabular cup scanned by XCT 


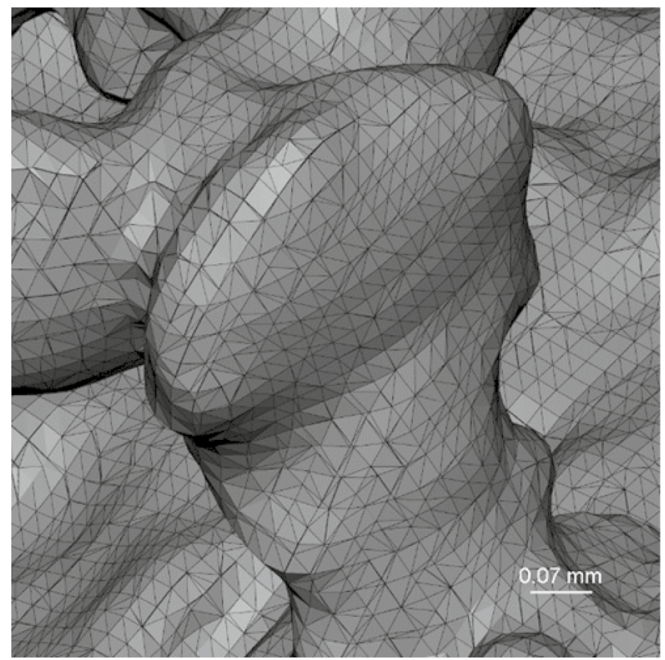

(a)

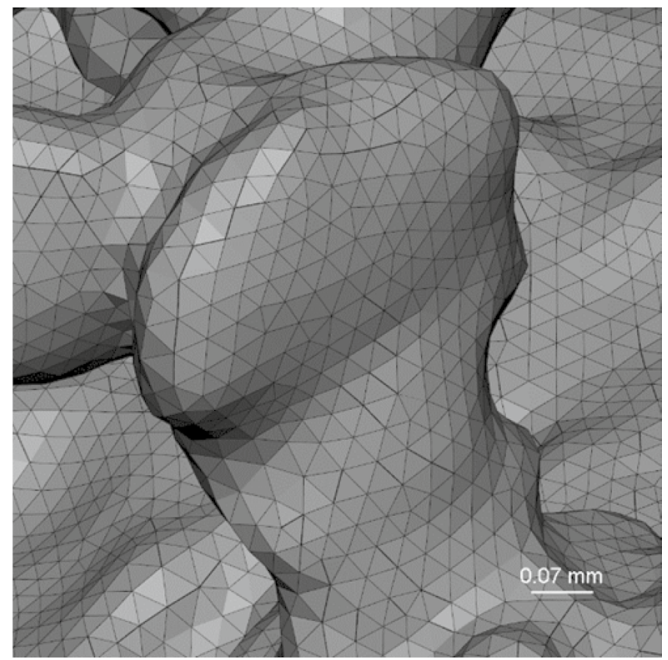

(b)

Fig. 5 Extracted and remeshed surface patch from the scaffold structure of acetabular cup: a portion of mesh extracted from the volume; $\mathbf{b}$ portion of mesh after repairing and remeshing

acetabular cup, the parameters per ISO 25178-2 [29] cannot be computed. Instead, the manifold parameters defined by Pagani et al. [30] are used to evaluate the surface texture on the cellular scaffold.

The initial step of the 3D surface characterisation is the computation of a reference form surface such that surface texture can be defined. The nominal CAD model is an option for the reference surface, if available. In the case that the CAD module is unknown, or using the nominal model would not be possible to measure the feature of interest due to large dimensional deviation, using an appropriate mathematical operator can be an alternative option to compute the reference surface. A feasible method to compute the reference form surface is using the morphological opening operator in order to enhance the peak of an additively manufactured surface [31]. In this study, the extracted surface is converted into a signed distance. The morphological opening operation is then performed on the 3D volume data. A voxel size of $25 \mu \mathrm{m}$ was set in the mesh-to-volume conversion, while an offset of $100 \mu \mathrm{m}$ was used in the morphological opening operator. The volume was processed using OpenVDB [32], a fast and efficient $\mathrm{C}++$ library designed to perform operations on sparse volume. The generated mesh was then manually cut into four surface patches, and each of the patches was individually analysed.

The second step of the characterisation process is the computation of the signed distance between the measured surface and the reference form surface. The distance has a positive value if the measured point is located along the direction of the outgoing normal of the reference surface, negative otherwise. The pseudo-normal method implemented in LibIGL [33] was used to compute the signed distance. A portion of the measured mesh and the computed reference form are shown in Fig. 6. The morphological opening operation resulted in a smooth reference surface with the globule generated by the AM process suppressed.

To compute the manifold parameters, each measured point projected to the reference surface has the correspondent signed distance. The height manifold parameters can be therefore computed as the integral of a scalar field on a surface. The mean absolute value of the height, root mean square height, skewness and kurtosis of the heights are reported below:

$$
\begin{aligned}
& \mathrm{Sa}=\frac{1}{A_{\text {form }}} \iint_{\Sigma_{\text {form }}}\left|r_{\mathrm{sl}}(u, v)\right| \mathrm{d} \sigma_{\text {form }} \\
& \mathrm{Sq}=\sqrt{\frac{1}{A_{\text {form }}} \iint_{\Sigma_{\text {form }}} r_{\mathrm{sl}}^{2}(u, v) \mathrm{d} \sigma_{\text {form }}} \\
& \mathrm{Ssk}=\frac{1}{A_{\text {form }} \cdot \mathrm{Sq}^{3}} \iint_{\Sigma_{\text {form }}} r_{\mathrm{sl}}^{3}(u, v) \mathrm{d} \sigma_{\text {form }} \\
& \mathrm{Sku}=\frac{1}{A_{\text {form }} \cdot \mathrm{Sq}^{4}} \int_{\Sigma_{\text {form }}} r_{\mathrm{sl}}^{4}(u, v) \mathrm{d} \sigma_{\text {form }} .
\end{aligned}
$$

where

$$
\mathrm{d} \sigma_{\text {form }}=r_{\text {form }, u}(u, v) \times r_{\text {form }, v}(u, v) \mathrm{d} u \mathrm{~d} v
$$

and $\boldsymbol{r}_{\text {form }, i}(u, v)$ is the partial derivative of $\boldsymbol{r}_{\text {form }}(u, v)$ in the $i$ direction, $\mathrm{d} \sigma_{\text {form }}$ is the infinitesimal areal element and $A_{\text {form }}=\iint_{\Sigma_{\text {form }}} \mathrm{d} \sigma_{\text {form }}$ is the area of the form surface. The esti- 


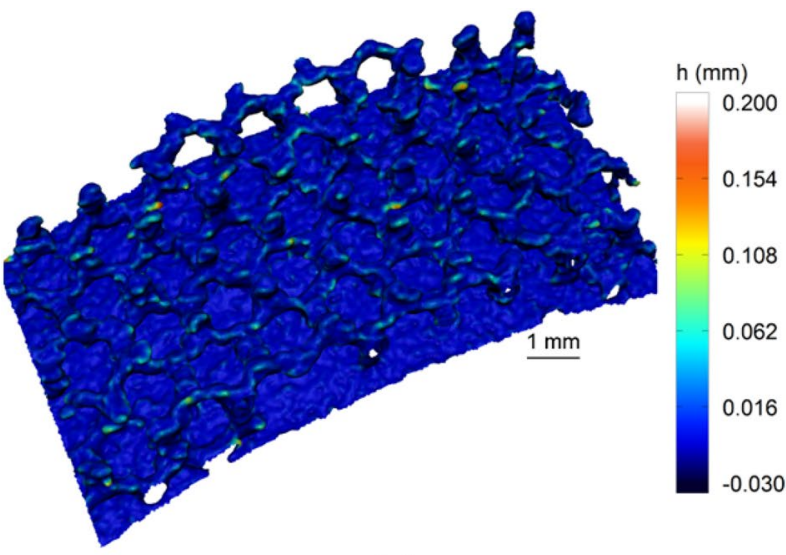

(a)

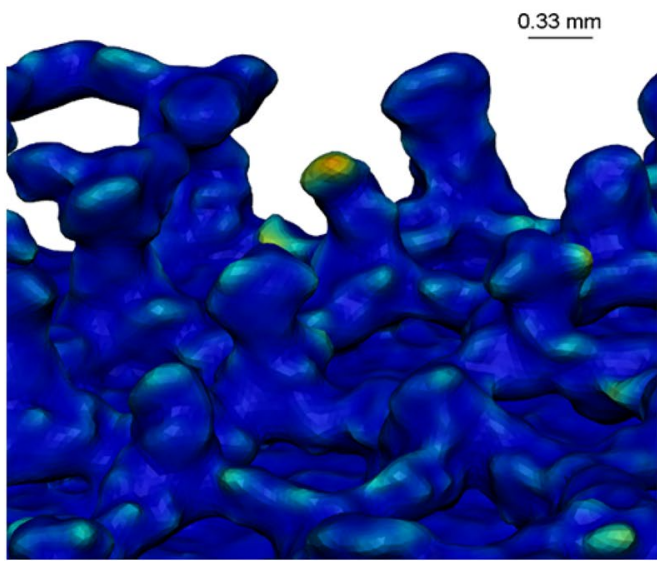

(c)

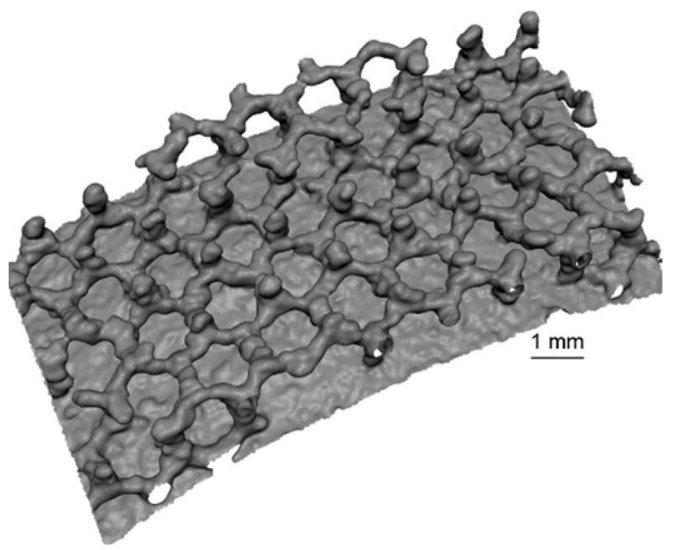

(b)

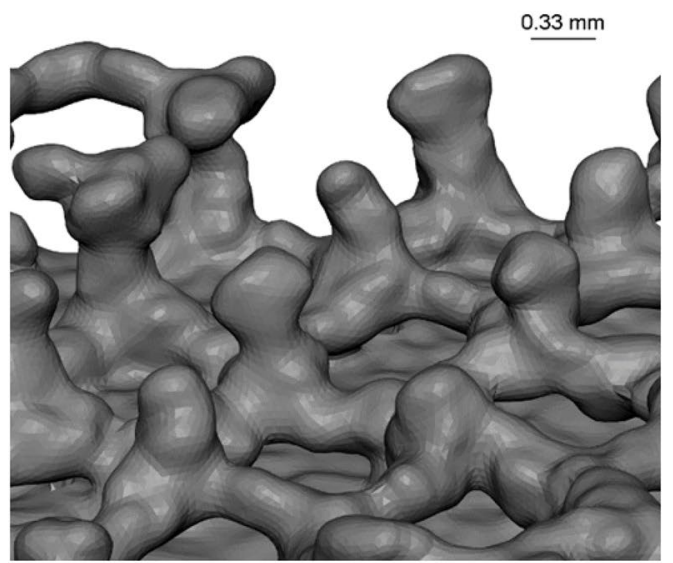

(d)

Fig. 6 Extracted mesh and reference surface (the colour represents the distance between the measured and the closest point of the reference surface): a measured surface; $\mathbf{b}$ reference form surface; $\mathbf{c}$ magnified portion of the measured surface; $\mathbf{d}$ magnified portion of the reference surface

mated surface texture parameters of the selection are listed in Table 2 and plotted in Fig. 7.

In addition to the height parameters listed in Table 2, another set of surface texture parameter that could be useful for the characterisation of the acetabular cup is the so-called feature parameters [34]. The surface topography is first segmented into a variety of local regions. Indexes for characterising the surface are then computed for each region. The watershed segmentation [35] is used to segment the mesh surface. Due to the local trivial surface topographical features as well as the measurement noises, the watershed segmentation tends to produce a number of tiny local segments, which is often called over-segmentation. This issue can be solved by building a change tree and applying the Wolf pruning to reduce the leaves with height differences less than a given threshold value [36]. An empirical value of $12 \mu \mathrm{m}$ is used to reduce the number of segmented regions. Two types of segmentation are adopted, one based on surface peaks and the other one based on surface pits. Both surface peaks and pits are closely related to cell proliferation. The segmented regions of surface pits and peaks are shown in Fig. 8.

Two straightforward parameters to characterise surface peaks and pits are the density of the peaks of the measured area
Table 2 Estimated surface manifold parameters along with the mean and the standard deviation of the analysed surface patches

\begin{tabular}{lrrrrrr}
\hline Surface patch no. & \multicolumn{1}{c}{1} & \multicolumn{1}{c}{3} & \multicolumn{1}{c}{4} & Mean & STD \\
\hline Sa $(\mu \mathrm{m})$ & 5.94 & 5.71 & 5.61 & 5.73 & 5.75 & 0.14 \\
Sq $(\mu \mathrm{m})$ & 10.77 & 10.33 & 9.94 & 11.62 & 10.67 & 0.72 \\
Ssk & 4.29 & 4.22 & 3.84 & 6.99 & 4.84 & 1.45 \\
Sku & 32.26 & 31.89 & 24.92 & 94.83 & 45.98 & 32.74 \\
\hline
\end{tabular}


Fig. 7 Estimated surface texture parameters of the selection. Each circle represents a sample surface patch, coloured according to its number. In the figures are shown the mean (red square) and its $95 \%$ confidence interval: a Sa; b Sq; c Ssk; d Sku

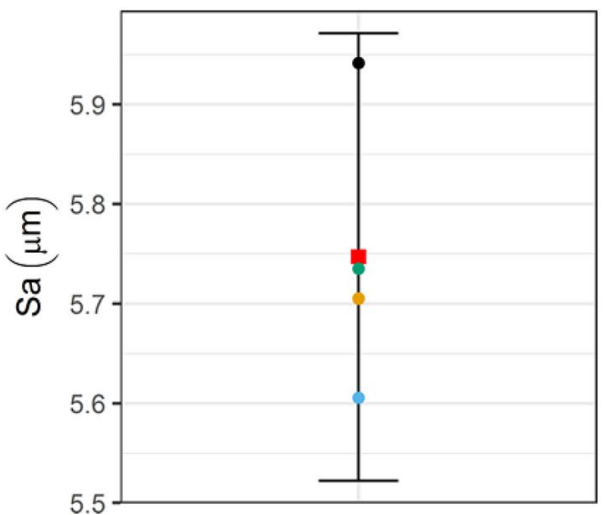

(a)

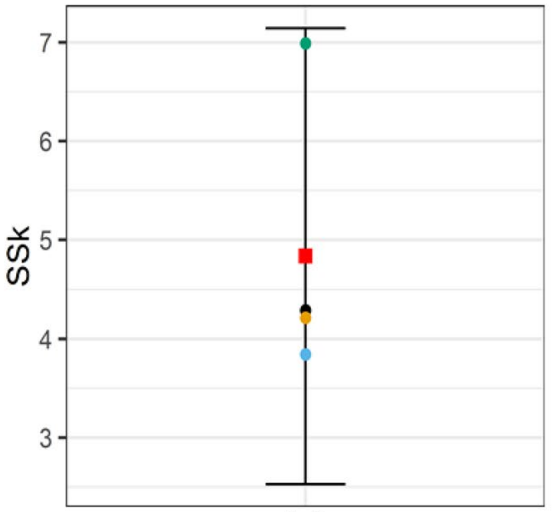

(c)

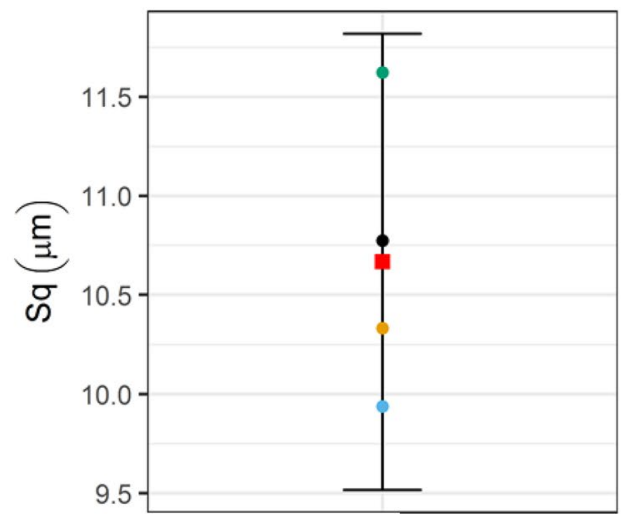

(b)

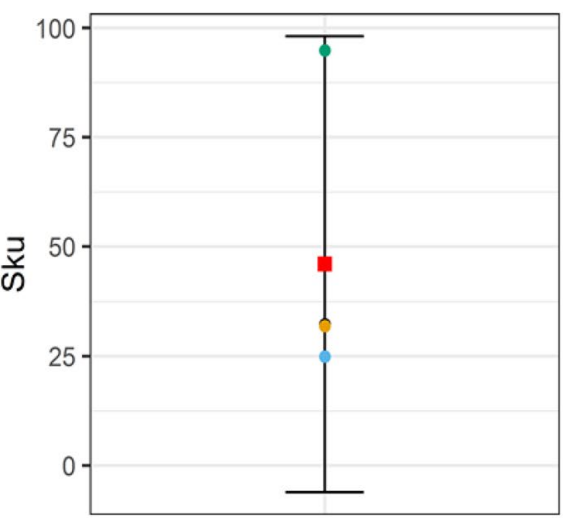

(d)
Spd $=\frac{\# \text { peaks }}{A}$

and the density of the pits (valleys)

Svd $=\frac{\# \text { valleys }}{A}$

where $A$ is the area of the analysed surface. Since each segmented region only contains a single significant peak/pit, the number of the peaks and pits corresponds to the number of the regions for peaks and pits, respectively. The estimated peak density and pit density are listed in Table 3 and plotted in Fig. 9.

\section{Discussion}

$\mathrm{XCT}$ is capable of capturing surface texture provided that it has the required resolution to resolve the fine details of surface texture. Considering the size of the acetabular cup used in this study, the X-ray source is placed close to the measured acetabular implant sample in order to obtain a maximised magnification and therefore a minimised voxel size. The experiment studies show that for a typical as-built
AM surface the voxel size for full characterisation should be less than one-half the surface Sa value [37]. The average value of resulted $\mathrm{Sa}$ of the measured acetabular cup is $5.75 \mu \mathrm{m}$, which is far smaller than the voxel size $31.7 \mu \mathrm{m}$. Even with the sub-voxel surface determination of VGStudio software, where one-tenth voxel size accuracy might be expected [21], the currently used voxel size is still far from satisfaction. To solve this issue, the acetabular cup shall be scanned by a more capable XCT system. Alternatively, the top section of the cup can be scanned in order to reduce the size of sample for scanning.

The 3D nature of the cellular scaffold of the acetabular implant makes surface roughness difficult to be evaluated because current areal surface texture parameters per ISO 25178-2 [29] are defined on a 2.5D basis. In terms of its applicability, two restrictions apply: measurement points should be uniformly sampled and surface texture is supposed to be measured on a planar or nearly planar basis [38]. The fatal issue of evaluating XCT measured surface texture, however, is that XCT measurement data does not conform to these two constraints as required by ISO 257182. Firstly, XCT scan data (represented by triangular meshes) does not comply with the uniform sampling requirements of traditional areal surface texture parameters; secondly, and more restricting, XCT captured data consist of 3D shapes 


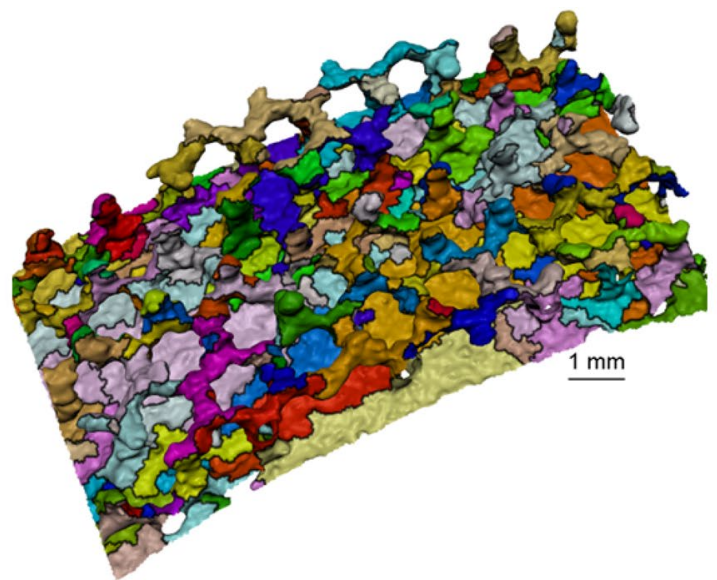

(a)

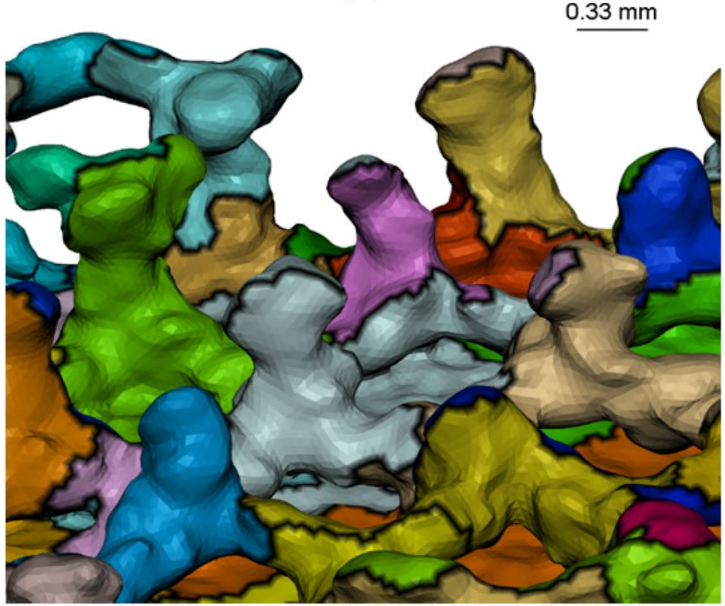

(c)

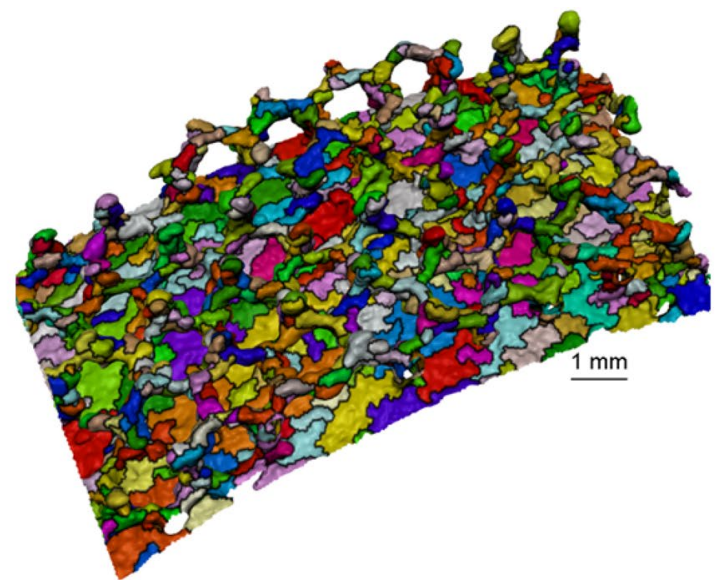

(b)

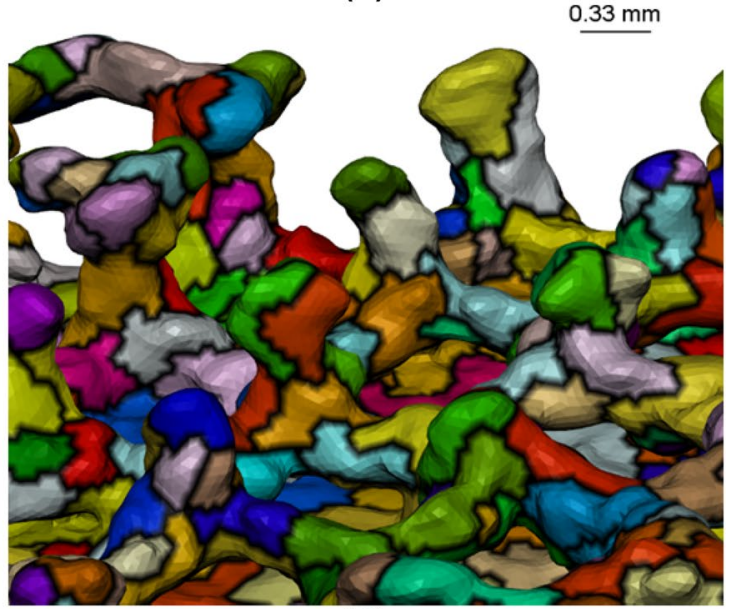

(d)

Fig. 8 Watershed segmentation of cellular scaffold surfaces with threshold value $12 \mu \mathrm{m}$ : a pit-based watershed segmentation; b peak-based watershed; $\mathbf{c}$ magnified portion of the pit-based watershed segmentation; $\mathbf{d}$ magnified portion of the peak-based watershed segmentation

Table 3 Estimated feature parameters along with the mean and the standard deviation of the analysed surface patches

Fig. 9 Estimated feature parameters and $95 \%$ mean confidence interval: a peak density; b pit density

\begin{tabular}{lllllll}
\hline Surface patch no. & 1 & 2 & 3 & 4 & Mean & STD \\
\hline Spd $\left(1 / \mathrm{mm}^{2}\right)$ & 0.22 & 0.24 & 0.23 & 0.23 & 0.23 & 0.01 \\
Svd $\left(1 / \mathrm{mm}^{2}\right)$ & 0.97 & 1.18 & 1.08 & 1.10 & 1.08 & 0.09 \\
\hline
\end{tabular}

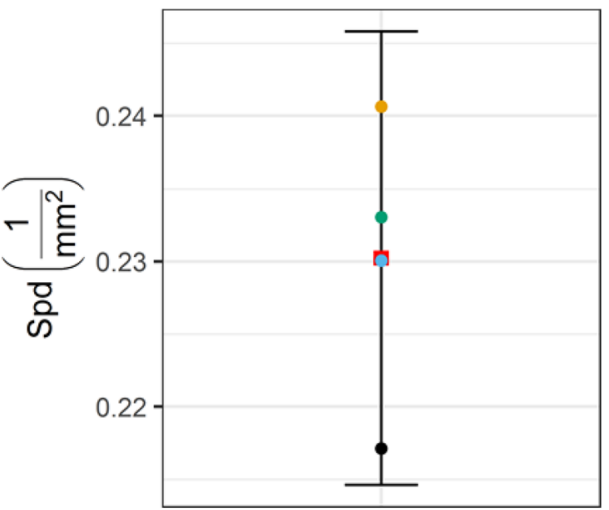

(a)

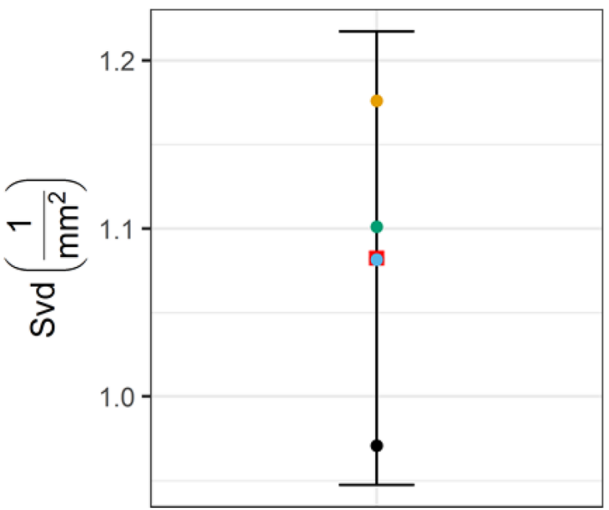

(b) 
of scaffold structure, which is impossible to be projected onto a $2 \mathrm{D}$ plane to enable the implementation of traditional areal parameters. The development of 3D manifold parameters provides a solution to solve the deadlock. The newly developed parameters extended traditional areal surface texture parameters into the $3 \mathrm{D}$ case, where surface texture is evaluated on the surface form without having to be projected onto a $2 \mathrm{D}$ plane. The form used in this study was estimated via morphological operation on volumetric data. Ideally, the nominal designed data, e.g. CAD, are opted if available. In this case, the XCT scanned data can be registered to the CAD to facilitate a better extraction of surface texture. On the contrary, the form estimation using the morphological approach can generate a smooth reference surface and facilitate the watershed segmentation of globule-like features.

The 3D height parameters, i.e. Sa, Sq, Sku, Ssk, give a quantitative evaluation of the surface quality of cellular scaffold. They can indicate the roughness and sharpness of the surface texture from a statistical point of view. The proposal of using feature-based parameters, e.g. peak density and pits density, provides a complimentary assessment of surface quality from the bio-functional aspect. These parameters are implemented based on the 3D watershed segmentation of surface topography features. They are closely related to the cell communication and tissue growth. There is a spectrum of feature-based parameters, such as arithmetic mean peak curvature, five-point pit height, mean dale area, mean hill volume, which might be more capable of linking surface quality of cellular scaffold with the implant osseointegration. This will be considered in future work.

The surface quality of cellular scaffold is evaluated based on each extracted triangular mesh surface patch. It, however, does not imply the restriction of having to cut the scaffold surface into pieces. Instead, it offers the flexibility of selecting any region of interest (ROI), no matter whether it is the integral surface or a local patch of the integral surface. The selection of ROI depends on the specific requirement, but the proposed methodology shall work in every case.

\section{Conclusion}

It is well known that open-cellular scaffold structures are effective structures to support tissue growth and boneimplant fixation. Surface texture of the scaffold is playing a major role in stimulating cell communication and proliferation. The measurement and characterisation of the scaffold's surface texture are proved to be very difficult due to its $3 \mathrm{D}$ nature. This paper proposes to use XCT to conduct a nondestructive measurement of the surface texture of an EBMproduced titanium acetabular cup with a cellular scaffold in-growth layer. The surface texture of 3D scaffold is evaluated using the newly developed 3D manifold parameters, which enables surface characterisation on triangular mesh surfaces. Four commonly used height parameters, i.e. Sa, Sq, Ssk, Sku, are resulted from four surface patches extracted from the XCT measured surface. In addition, the surface peak density and pit density are estimated based on the 3D watershed segmentation. The Wolf pruning with an empirical threshold value is used to control the over-segmentation. These two feature parameters are believed to be more relevant to cell growth. While the proposed methodology can provide a feasible solution for the evaluation of the surface quality of cellular scaffold, some improvements are suggested in order to achieve a more accurate assessment of scaffold surface texture, e.g. capturing data with better XCT resolution/smaller voxel size, and using nominal design data to extract surface texture. These will be considered in future work.

Acknowledgements The authors gratefully acknowledge the UK's Engineering and Physical Sciences Research Council (EPSRC) funding of the Future Advanced Metrology Hub (EP/P006930/1). S. Lou would like to thank the EPSRC (EP/S000453/1) for funding this work.

\section{Compliance with ethical standards}

Conflict of interest The authors declare that they have no conflict of interest

Ethical approval This paper does not contain any studies with human or animal subjects.

Open Access This article is distributed under the terms of the Creative Commons Attribution 4.0 International License (http://creativeco mmons.org/licenses/by/4.0/), which permits unrestricted use, distribution, and reproduction in any medium, provided you give appropriate credit to the original author(s) and the source, provide a link to the Creative Commons license, and indicate if changes were made.

\section{References}

1. Tan XP, Tan YJ, Chow CSL, Tor SB, Yeong WY (2017) Metallic powder-bed based 3D printing of cellular scaffolds for orthopaedic implants: a state-of-the-art review on manufacturing, topological design, mechanical properties and biocompatibility. Mater Sci Eng C 76:1328-1343

2. de Vasconcellos LM, Cairo CA, de Vasconcellos LG, de Alencastro Graça ML, do Prado RF, Carvalho YR (2012) Porous titanium, by powder metallurgy for biomedical application: characterization, cell citotoxity and in vivo tests of osseointegration. INTECH Open Access Publisher, Thousand Oaks

3. Biemond JE, Eufrasio TS, Hannink G, Verdonschot N, Buma P (2011) Assessment of bone in-growth potential of biomimetic hydroxyapatite and brushite coated porous E-beam structures. J Mater Sci Mater Med 22(4):917-925

4. Li X, Feng YF, Wang CT, Li GC, Lei W, Zhang ZY, Wang L (2012) Evaluation of biological properties of electron beam melted Ti6A14V implant with biomimetic coating in vitro and in vivo. PLoS ONE 7(12):e52049 
5. Singh R, Singh S, Kapoor P (2016) Investigating the surface roughness of implant prepared by combining fused deposition modeling and investment casting. J Process Mech Eng 230(5):403-410

6. Hutmacher DW (2000) Scaffolds in tissue engineering bone and cartilage. Biomaterials 21:2529-2543

7. Harrysson OLA, Cansizoglu O, Marcellin-Little DJ, Cormier DR, West HA (2008) Direct metal fabrication of titanium implants with tailored materials and mechanical properties using electron beam melting technology. Mater Sci Eng C 28:366-373

8. Novaes AB Jr, Souza SL, Barros RR, Pereira KK, Iezzi G, Piattelli A (2010) Influence of implant surfaces on osseointegration. Braz Dent J 21(6):471-481

9. Hansson S, Norton M (1999) The relation between surface roughness and interfacial shear strength for bone-anchored implants. A mathematical model. J Biomech 32:829-836

10. Biemond JE, Hannink G, Verdonschot N, Buma PJ (2013) Bone ingrowth potential of electron beam and selective laser melting produced trabecular-like implant surfaces with and without a biomimetic coating. Mater Sci Mater Med 24:745-753

11. de Wild M, Schumacher R, Mayer K, Schkommodau E, Thoma D, Bredell M, Kruse Gujer A, Grätz KW, Weber FE (2013) Bone regeneration by the osteoconductivity of porous titanium implants manufactured by selective laser melting: a histological and micro computed tomography study in the rabbit. Tissue Eng 19:2645-2654

12. Jalota S, Bhaduriand SB, Tas AC (2007) Osteoblast proliferation on neat and apatite-like calcium phosphate-coated titanium foam scaffolds. Mater Sci Eng C 27:432-440

13. Sing SL, An J, Yeong WY, Wiria FE (2015) Laser and electronbeam powder-bed additive manufacturing of metallic implants: a review on processes, materials and designs. J Orthop Res 34:369-385

14. Ponader S, Vairaktaris E, Heinl P, Wilmowsky CV, Rottmair A, Körner C, Singer RF, Holst S, Schlegel KA, Neukam FW, Nkenke E (2008) Effects of topographical surface modifications of electron beam melted Ti-6Al-4V titanium on human fetal osteoblasts. J Biomed Mater Res Part A 84:1111-1119

15. Boyan BD, Dean DD, Lohmann CH, Cochran DL, Sylvia VL, Schwartz Z (2001) The titanium-bone cell interface in vitro: the role of the surface in promoting osteointegration. In: Brunette DM, Tengvall P, Textor M, Thomsen P (eds) Titanium in medicine. Springer, Berlin, pp 561-586

16. Lou S, Sun W, Brown B, Pagani L, Zeng W, Jiang X, Scott PJ (2018) Simulation for XCT and CMM measurements of additively manufactured surfaces, ASPE and euspen summer topical meeting advancing precision in additive manufacturing, Lawrence Berkeley National Laboratory, Berkeley, California, USA

17. Kruth JP, Bartscher M, Carmignato S, Schmitt R, De Chiffre L, Weckenmann A (2011) Computed tomography for dimensional metrology. CIRP Ann Manuf Technol 60:821-842

18. Weckenmann A, Kramer P (2013) Computed tomography in quality control: chances and challenges. Proc Inst Mech Eng B J Eng 227:634-642

19. Borges de Oliveira F, Stolfi A, Bartscher M, De Chiffre L, Neuschaefer-Rube U (2016) Experimental investigation of surface determination process on multi-material components for dimensional computed tomography. Case Stud Nondestr Test Eval B 6:93-103
20. Lifton JJ, Malcolm AA, McBride JW (2015) On the uncertainty of surface determination in X-ray computed tomography for dimensional metrology. Meas Sci Technol 26:035003

21. Reinhart C (2011) Industrial CT \& precision, conference in "Application of CT Scanning in Industry", Heidelberg, Germany

22. Lorensen WE, Cline HE (1987) Marching cubes: a high resolution 3D surface construction algorithm. Comput Gr 21(4):163-169

23. Kerckhofs G, Pyka G, Moesen M, van Bael S, Schrooten J, Wevers M (2012) High-resolution microfocus X-ray computed tomography for 3D surface roughness measurements of additive manufactured porous materials. Adv Eng Mater 15:153-158

24. Townsend A, Pagani L, Scott P, Blunt L (2017) Areal surface texture data, extraction from X-ray computed tomography reconstructions of metal additively manufactured parts. Precis Eng 48:254-264

25. Thompson A, Senin N, Giusca C, Leach R (2017) Topography of selectively laser melted surfaces: a comparison of different measurement methods. Ann CIRP 66:543-546

26. Volume Graphics GmbH, VGStudio MAX

27. Centin M, Signoroni A (2015) RameshCleaner: conservative fixing of triangular meshes. Smart tools and apps for graphics-Eurographics Italian chapter conference, The Eurographics Association

28. Botsch M, Kobbelt L, Pauly M, Alliez P, Levy B (2010) Polygon mesh processing. Taylor \& Francis, Thames

29. ISO 25178-2 (2012) Geometrical product specifications (GPS) surface texture: areal-part 2: terms, definitions and surface texture parameters

30. Pagani L, Qi QF, Jiang X, Scott PJ (2017) Towards a new definition of areal surface texture parameters on freeform surface. Measurement 109:281-291

31. Lou S, Jiang X, Sun W, Zeng W, Pagani L, Scott PJ (2018) Characterisation methods for powder bed fusion processed surface topography. Precis Eng. https://doi.org/10.1016/j.precisione ng.2018.09.007

32. Museth K, Lait J, Johanson J, Budsberg J, Henderson R, Alden M, Cucka P, Hill D, Pearce A (2013) OpenVDB: an open-source data structure and toolkit for high-resolution volumes. Proc ACM SIGGRAPH Courses 20(10):1405-1417

33. Jacobson A, Panozzo D et al (2016) LIBIGL: a simple C++ geometry processing library. http://libigl.github.io/libigl/. Accessed 5 Apr 2019

34. Scott PJ (2009) Feature parameters. Wear 266:548-551

35. Mesmoudi M, De Floriani L (2007) Morphology-based representations of discrete scalar fields. In: GRAPP 2007, Barcelona, Spain, pp 137-144

36. Scott PJ (2004) Pattern analysis and metrology: the extraction of stable features from observable measurements. Proc R Soc A 460:2845-2864

37. Townsend A, Racasan R, Leach R, Senin N, Thompson A, Ramsey A, Bate D, Woolliams P, Brown S, Blunt L (2018) An interlaboratory comparison of X-ray computed tomography measurement for texture and dimensional characterisation of additively manufactured parts. Addit Manuf 23:422-432

38. Abdul-Rahman H, Lou S, Zeng W, Jiang X, Scott PJ (2016) Freeform texture representation and characterisation based on triangular mesh parameterisation techniques. Measurement 92:172-182 